\title{
Clinical Phenotype of LRRK2 R1441C in 2 Chinese Sisters
}

\author{
Shen-Yang Limª, Jia Lun Limº, Azlina Ahmad-Annuarc Katja Lohmann ${ }^{\text {d }}$ \\ Ai Huey Tan ${ }^{a, b}$ Kai Bin Lima,b Yi Wen Tayc Yee Lee Shing ${ }^{\mathrm{e}}$ \\ Kalai Arasu Muthusamy ${ }^{f}$ Peter Bauerg Arndt Rolfs ${ }^{g}$ Christine Klein ${ }^{d}$ \\ ${ }^{a}$ Division of Neurology, Faculty of Medicine, University of Malaya, Kuala Lumpur, Malaysia; ${ }^{\mathrm{b}}$ The Mah Pooi Soo \\ and Tan Chin Nam Centre for Parkinson's and Related Disorders, University of Malaya, Kuala Lumpur, Malaysia;

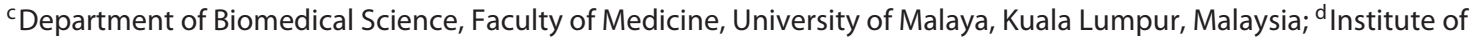 \\ Neurogenetics, University of Lubeck, Lubeck, Germany; ${ }^{e}$ Department of Psychology, Goethe University Frankfurt, \\ Frankfurt am Main, Germany; ${ }^{\mathrm{f} D i v i s i o n}$ of Neurosurgery, Faculty of Medicine, University of Malaya, Kuala Lumpur, \\ Malaysia; ${ }^{9}$ Centogene AG, Rostock, Germany
}

\section{Keywords}

Parkinson's disease $\cdot L R R K 2 \cdot \mathrm{R} 1441 \mathrm{C} \cdot$ Asian subjects .

Genetics · CISI-PD · Rating scales · Deep brain stimulation

\section{Abstract}

Pathogenic and risk variants in the LRRK2 gene are among the main genetic contributors to Parkinson's disease (PD) worldwide, and LRRK2-targeted therapies for patients with PARK-LRRK2 are now entering clinical trials. However, in contrast to the LRRK2 G2019S mutation commonly found in Caucasians, North-African Arabs, and Ashkenazi Jews, relatively little is known about other causative LRRK2 mutations, and data on genotype-phenotype correlations are largely lacking. This report is from an ongoing multicentre study in which next-generation sequencing-based PD gene panel testing has so far been conducted on 499 PD patients of various ethnicities from Malaysia. We describe 2 sisters of Chinese ancestry with PD who carry the R1441C mutation in LRRK2 (which in Asians has been reported in only 2 Chinese patients previously), and highlight interesting clinical observations made over a decade of close follow-up. We further

$\begin{aligned} & \text { karger@karger.com } \\ & \text { www.karger.com/ndd }\end{aligned}$
Karger $\%$

explored the feasibility of using a brief, expert-administered rating scale (the Clinical Impression of Severity Index; CISIPD) to capture data on global disease severity in a large ( $n=$ 820) unselected cohort of PD patients, including severely disabled individuals typically excluded from research studies. All patients in this study were managed and evaluated by the same PD neurologist, and these data were used to make broad comparisons between the monogenic PD cases versus the overall "real world" PD cohort. This report contributes to the scarce literature on R1441C PARK-LRRK2, offering insights into natural history and epidemiological aspects, and provides support for the application of a simple and reliable clinical tool that can improve the inclusion of underrepresented patient groups in PD research.

(c) 2020 S. Karger AG, Basel

Shen-Yang Lim

Neurology Laboratory, Level 6 South Block, University of Malaya Medical Centre 50603 Kuala Lumpur (Malaysia)

limshenyang@gmail.com

Christine Klein

Institute of Neurogenetics, University of Luebeck

Ratzeburger Allee 160, DE-23538 Luebeck (Germany)

christine.klein@ neuro.uni-luebeck.de

Azlina Ahmad-Annuar

Department of Biomedical Science, Faculty of Medicine

University of Malaya, 50603 Kuala Lumpur (Malaysia)

azlina_aa@um.edu.my 


\section{Introduction}

LRRK2 gene mutations and variants are among the main genetic contributors to Parkinson's disease (PD) worldwide $[1,2]$. Genetics-based therapeutic approaches targeted at LRRK2 have now started to enter clinical trials and are primarily centred on inhibition of LRRK2 kinase activity $[3,4]$. Indeed, there is abundant literature showing that the most common LRRK2 mutation found in Caucasians, G2019S, results in augmented LRRK kinase activity $[3,4]$. However, this causative autosomal dominant mutation is exceedingly rare in all Asian $\mathrm{PD}$ populations studied so far [5, 6]. Instead, "Asian $L R R K 2$ risk variants" such as G2385R and/or R1628P, which are associated with approximately a 2-fold elevated risk of developing PD, are common and present in up to $5-10 \%$ of East Asian (Chinese, Japanese, Korean) patients [58]. The molecular mechanisms of these Asian risk variants contributing to PD remain largely undefined $[4,6]$.

Other causative autosomal dominant LRRK2 mutations (e.g., involving substitution of Arginine at position 1,441 with cysteine, glycine, or histidine) appear to be rare worldwide, with the exception of the R1441G mutation underlying a large proportion of familial PD cases in the Spanish Basque population [4, 9]. Only 2 Asian cases of R1441C PARK-LRRK2 have been reported previously [10-14], and further study is needed as many under-represented populations have not been tested $[6,15,16]$. Moreover, since the preponderance of studies have focused on the G2019S mutation $[4,13]$, there is a need for further genotype-phenotype characterisation of these rarer monogenic cases [17]. For example, out of a total of 38 patients harbouring the $\mathrm{R} 1441 \mathrm{C}$ mutation in the MDSGene Database [13], which collates all cases worldwide reported on the individual level published in English, the presence or absence of cognitive decline and psychosis was reported in only 12 and 5 patients, respectively [14].

Herein, we provide detailed descriptions of 2 Malaysian sisters of Chinese ancestry heterozygous for the LRRK2 p.R1441C (c.4321C >T) mutation, 1 of whom had a fairly "typical" presentation (but required device-aided therapies to control motor complications), and the other who had some atypical features. The mutations were detected as part of an ongoing multicentre study in which 499 PD patients of various ethnicities from Malaysia have undergone testing using a next-generation sequencing (NGS)-based PD gene panel [2]. As an exploratory method to gauge the clinical severity of our monogenic PD cases against that of a large unselected cohort, we charted their 10-year disease trajectory and compared this with cross-sectional data from 820
PD patients (all patients managed and rated by the same Parkinson's neurologist; S.-Y.L.).

\section{Materials and Methods}

\section{Genotyped Patients}

The study was approved by the Medical Research Ethics Committees, University of Malaya Medical Centre (UMMC) and Ministry of Health Malaysia. Written informed consent was obtained from all subjects. Patients with PD were recruited from five tertiary centres in Malaysia. Diagnosis of PD was assigned by neurologists according to standard clinical diagnostic criteria (Queen Square Brain Bank criteria, but not including the presence of family history as an exclusion criterion) [18]. Recruitment was open to all consenting adult patients, with an emphasis on those with younger onset and/or positive family history (convenience sampling). Four hundred and ninety-nine patients (56.9\% males, $43.1 \%$ females) were analysed for this study, with a mean age at PD diagnosis of $56.3 \pm 13.2$ years (range 16-86). Overall, $14.6 \%(73 / 499)$ and $33.1 \%$ $(165 / 499)$ had an age of PD diagnosis $\leq 40$ and $\leq 50$ years, respectively, and $18.2 \%(91 / 499)$ had a family history of either confirmed or possible PD. The clinical characteristics of PD patients with causative LRRK2 mutations (PARK-LRRK2) were sourced from patients' charts and other prospectively collected materials.

\section{Genetic Analysis}

All subjects underwent testing using an NGS-based PD gene panel (Parkinson panel at Centogene AG, Rostock, Germany) which included all exons of PRKN, PINK1, DJ-1, SNCA, LRRK2, GBA, VPS35, PLA2G6, RAB39B, and exons 11, 37, 43, 61, and 69 of $V P S 13 C$ [2].

Comparison of Clinical Data Including CISI-PD from a Large Local Cohort of Patients

Basic demographic and clinical data were obtained from consecutive patients attending routine consultations in the neurology clinics at UMMC (public clinic) and UMSC (University of Malaya Specialist Centre, a private clinic), from February 20, 2019 to March 18, 2020. Although UMMC is a tertiary centre, a large proportion of PD patients are self-referred from the community. Ratings of clinical PD global severity were performed by a single experienced Parkinson's neurologist (S.-Y.L.), blinded to the patients' genetic status, using the Clinical Impression of Severity Index-PD for PD (CISI-PD) scale [19-21]. The CISI-PD encompasses four domains (parkinsonian motor signs, disability, motor complications, and cognitive status), each rated on a $0-6$ Likert scale giving a range of total score from 0 to 24 (higher scores indicating worse severity). This scale was previously demonstrated to correlate well with disease duration, measures of PD manifestations, disability, quality of life, and with other global severity scales $[19,20]$.

\section{Results}

\section{Genetic Findings}

A known pathogenic heterozygous $L R R K 2$ variant was detected in only 2 out of 499 patients $(0.4 \%$; c. $4321 \mathrm{C}>\mathrm{T}$,
Lim et al. 
p.R1441C), who were sisters, and verified using Sanger sequencing. Neither sister carried any other rare (gno$\mathrm{mAD}$ minor allele frequency $<0.01$ ) protein-changing variant in the genes targeted by the gene panel. Online supplementary Figure 1 (for all online suppl. material, see www.karger.com/doi/10.1159/000508131) shows their sequence electropherograms. Furthermore, exon rearrangements in PRKN, PINK1, PARK7, and SNCA were excluded by multiplex ligation-dependent probe amplification (MLPA) analysis using the P051 kit from MRC Holland.

\section{Clinical Findings of R1441C Patients}

The clinical details of these 2 patients are described below. They have both been under regular follow-up by S.-Y.L. for $>10$ years. There were 11 other siblings in the family, including an older brother who was said to have died of "old age" but had "trembles" and movement difficulties in the final 2 years of his life (see online suppl. Fig. 2 for the family pedigree). Their parents were nonconsanguineous; the mother was also said to have died from "old age" but she may have had some difficulty with gait and balance; her eldest brother may have had PD. No other members of the extended family were known to have PD or other neurodegenerative disorders.

\section{Case Report of Patient 1}

This patient was a 77-year-old woman who was diagnosed with PD aged 37 years. She underwent staged bilateral pallidotomy in China at the age of 57 years, with improvement in dyskinesias. When first seen by us at age 65 years, her parkinsonism was moderate-to-severe (Unified PD Rating Scale [UPDRS] part III score 48.5 [range 0-108]). She ambulated independently using a walking frame without falls. There was a moderate right laterocollis and severe rigidity at the neck. Her Montreal Cognitive Assessment (MoCA) score at age 69 years was $24 / 30$. A checklist of motor and non-motor features is provided in online supplementary Table 1 . Her treatments consisted of levodopa/carbidopa (100/25 mg; 2 tablets t.i.d.), baclofen (20 mg t.i.d.) for neck stiffness, zolpidem (20 mg noct.) for insomnia, and slow-release levodopa-benserazide (100/25 mg; 2 capsules noct.), which have remained quite stable over time. At the age of 75 years, her motoric features were very advanced; her total CISI-PD score (performed blinded to the patient's genetic result) was 16 (motor signs 6 , disability 6 , motor complications 0 , cognitive status 4 ). Besides her very long disease duration, another remarkable feature has been the occurrence of "purposeless groaning," a phenomenon typically seen in advanced stages of progressive supranuclear palsy (PSP) and very rarely reported in PD [22-24]. This patient did not exhibit signs of PSP (e.g., impairment of vertical eye movements).

\section{Case Report of Patient 2}

PD was diagnosed at the age of 58 years, with a fairly "typical" course. Her initial symptom was a reduced arm swing on the right. Her only medical history at this time was hypertension; there was no history of exposure to environmental toxins. When first seen by us aged 63 years, she had mild-to-moderate severity motor fluctuations (medication effect lasting 4-4.5 h) without dyskinesias; her UPDRS part III score (performed as the patient was already wearing off, $4 \mathrm{~h} 45 \mathrm{~min}$ post-levodopa dosing) was 23 (mild-to-moderate impairment), with features more pronounced on the right side. Her OFF state was characterised by slowness and gait difficulty, whereas she felt "normal" in the ON state. She took levodopa/benserazide (200/50 mg; half a tablet 3-4 times daily) and ropinirole (8 $\mathrm{mg}$ daily). Over time, OFF periods became increasingly troublesome with additional symptoms (painful foot dystonia and tremors; overnight/morning akinesia) and ON periods shorter (2-3 h only), with partial benefit from progressive addition of entacapone, slow-release levodopa/ benserazide, and rotigotine patch (10 $\mathrm{mg}$ daily). Sleep became increasingly fragmented requiring treatment with low-dose clonazepam. Eight years into her disease, she was on a high dose of PD medication (levodopa equivalent daily dose [LEDD] approx. 1,650 mg/day), and continuous subcutaneous apomorphine infusion was initiated and uptitrated to $10 \mathrm{mg} / \mathrm{h}(150 \mathrm{mg} /$ day $)$ given during waking hours. She reported marked improvement in OFF time but had troublesome nocturnal bradykinesia and stiffness. Due to the inconvenience of daily infusions, she opted for deep brain stimulation (DBS). A 3-T brain MRI was unremarkable and bilateral subthalamic nucleus (STN) electrodes were implanted at age 67 years. During the initial programming (14 h OFF-medication), acute stimulation at the left STN with $2.5 \mathrm{~V}$ (single monopolar, $60 \mu \mathrm{s}, 130 \mathrm{~Hz}$ ) resulted in $100 \%$ improvement of rigidity at the right wrist (improvement with right STN stimulation was difficult to gauge as rigidity and tremor were minimal on the left side of the body at baseline). Stimulation-induced dyskinesias occurred in the ensuing days with $1.5 \mathrm{~V}$ bilaterally, suggesting correct target localisation of the DBS electrodes within the STN. LEDD was initially reduced to $650 \mathrm{mg} /$ day but had to be gradually increased to approximately $1,100 \mathrm{mg} /$ day by 1 year post-DBS. She had persistent troublesome OFF periods (ON periods lasting 3-4 h) and mild-to-moderate dyskinesias, despite numerous attempts at optimis- 


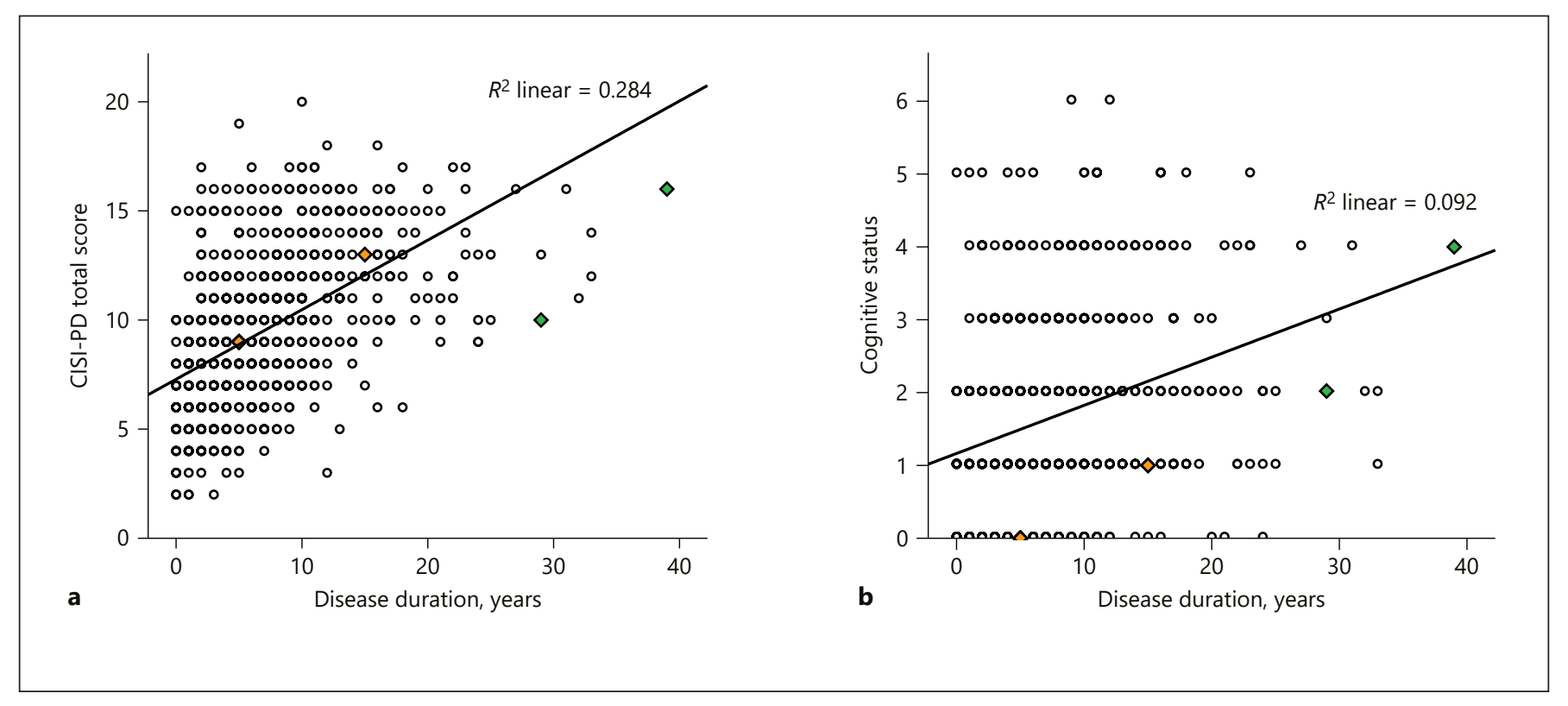

Fig. 1. Scatter plots showing CISI-PD total scores (a; range 0-24, higher scores indicating worse severity) and item 4 (cognitive status) subscores (b; range 0-6), obtained cross-sectionally from 820 PD patients, versus duration since PD diagnosis. Lines of best fit are shown. Patient 1 is represented by the green diamonds and Patient 2 by the orange diamonds. The following CISI-PD total scores have been suggested for classification of PD severity: 1-7 (mild), 8-14 (moderate), and 15-24 (severe) [19-21].

ing stimulation parameters. She also developed mild-tomoderate slurring of speech. At age 69 years ( 11 years postdiagnosis), a variety of clinical rating scales were administered as part of a study on body composition [25]: $\mathrm{ON}$-medication and ON-stimulation, the MDS-UPDRS part III score was 29 (range 0-132) indicating mild-tomoderate impairment [21]; part I, 9 points (range $0-52$ ) indicating a relatively mild non-motor symptom burden; part II, 7 points (range $0-52$ ) indicating mild functional disability, and MoCA 30/30. Her body mass index was 24.1 (pre-obese [26]). Occasional falls due to imbalance when turning occurred approximately 12 years after diagnosis. Her DBS battery lasted approximately 4 years and was replaced at age 71 years with a rechargeable device since she was thought to have a good medium-to-long-term outlook. Unfortunately, she developed gastric adenocarcinoma and interstitial lung disease at age 73 years and died the same year from cancer treatment-related septic complications. Four months prior to her demise (15 years of PD), her CISI-PD scores (conducted blinded to the patient's genetic result) were $4 / 4 / 4 / 1$, indicating "moderate" global PD severity [19-21], with a good cognitive status. Her moderate motor impairments were likely due in part to the cancer and its treatment.

\section{Comparison Cohort and CISI-PD Data}

The CISI-PD was completed for 820 patients, with a mean age of $67.1 \pm 10.2$ years (range 34-95), age at PD diagnosis $60.2 \pm 11.0$ years (range 23-90), disease duration $6.8 \pm 5.6$ years (range $0-39$ ), and LEDD $501.7 \pm 365.3$ $\mathrm{mg} /$ day (range 0-2,098.5). Patients of Chinese ethnicity comprised $78.7 \%$ of the cohort, Indians $13.7 \%$, and Malays 7.0\%. Sixty-three patients (7.7\%) had undergone DBS surgery and optimisation of their DBS parameters, and 8 $(1.0 \%)$ were treated with apomorphine infusion. The mean CISI-PD score was $9.5 \pm 3.4$ (range 2-20).

Figure 1 depicts the cross-sectional CISI-PD data plotted against duration since PD diagnosis, with the 2 R1441C patients' scores highlighted by coloured symbols. The availability of detailed clinic notes including complete UPRDS part III ratings (also by S.-Y.L.) from 2009 for the 2 sisters permitted CISI-PD scores to be retrospectively extrapolated for 2009 (4/4/0/2 and 3/3/3/0 points, respectively), to give a picture of their disease trajectory over a 10 -year period. The total CISI-PD scores in Figure 1a for Patient 1 are lower than the line of best fit, suggesting lower global PD severity than the "average" patient, whereas the scores for Patient 2 approximated the line of best fit, which was likely due to the counterbalancing of good cognitive status versus disabling motor response
Lim et al. 


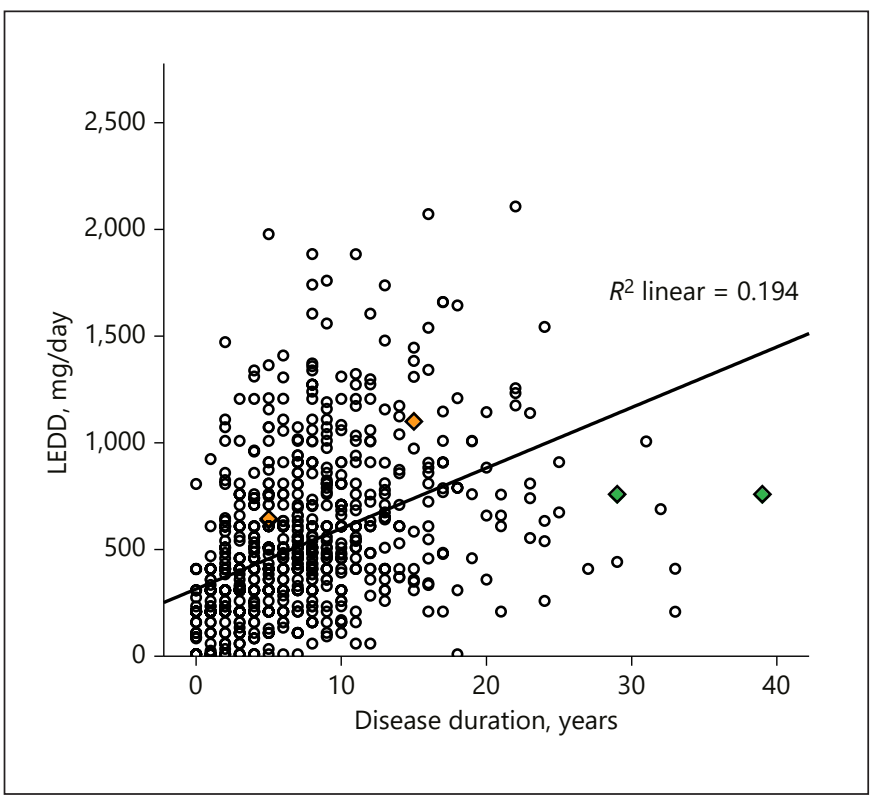

Fig. 2. Scatter plot showing dopaminergic medication requirement (LEDD) versus duration since PD diagnosis, obtained cross-sectionally from 820 PD patients. The line of best fit is shown. Patient 1 is represented by the green diamonds and Patient 2 by the orange diamonds.

complications. Figure $1 \mathrm{~b}$ shows that their cognitive status was better preserved compared to that of the overall cohort. Figure 2 depicts the patients' LEDDs (calculated for the same timepoint when CISI-PD was scored), plotted against disease duration; the relatively high LEDD for Patient 2 was driven by the need to control OFF periods.

\section{Discussion}

The clinical presentation of R1441C PARK-LRRK2 has previously been described to be "similar to" or "indistinguishable from" idiopathic PD [10-12, 27]. However, in reporting data from MDSGene, a high frequency of motor fluctuations was found in $\mathrm{R} 1441 \mathrm{C} / \mathrm{G} / \mathrm{H}$ patients (affecting $88 \%$ of patients vs. $65 \%$ of G2019S patients, for example) [13]. This is remarkable given that both our patients received "advanced" therapies (functional neurosurgery and/ or infusion of dopaminergic medication) to treat their motor complications, compared to approximately $10 \%$ of our overall patient cohort; Patient 2 also required a relatively high LEDD (Fig. 2) despite undergoing successful DBS surgery, for control of OFF periods. Systematic reviews of response to DBS among monogenic PD patients highlighted possible poorer outcomes in patients harbouring the LRRK2
R1441G mutation, but this was based on only a few cases with a limited duration of follow-up, and to our knowledge there are no reports in $\mathrm{R} 1441 \mathrm{C}$ patients $[28,29]$. The relatively young ages of onset of our cases (37 and 58 years) were also in keeping with MDSGene data - an updated search of R1441C PARK-LRRK2 revealed a median PD onset age of 58 years (range $30-78$ ). This contrasts with the preponderance of idiopathic PD cases where onset is usually later, in the mid-60s and beyond [30, 31]. Data regarding non-motor features in R1441C are very scarce, but there is a suggestion in the literature that problems such as dementia may be quite uncommon (reported in only a handful of cases so far) $[11,12,14]$. Although limited conclusions can be made based on only 2 patients, the findings in our study were consistent, with cognitive abilities remaining relatively intact during the disease course for 15 years or more. The very long (40-year) disease duration for Patient 1 was also noteworthy; so far, she has the longest PD duration in our cohort of approximately 1,500 PD patients (unpubl. data; a large international multicentre study focusing on long-term survivors documented only 5 patients living $\geq 40$ years with PD [32]).

A pertinent question is whether there are distinct disease mechanisms related to the R1441C mutation, and how these may correlate with the clinical phenotype. As summarised in a recent review (see Table 1 and Fig. 2 of Tolosa et al. [4]), the R1441 mutations are located in the LRRK2 Ras-of-complex (Roc) GTPase domain adjacent to the kinase domain and, like G2019S, are thought to cause a toxic gain-of-function of the LRRK2 kinase. However, animal models suggested that the R1441C mutation, compared to G2019S, may have a more profound effect on striatal physiology, with higher phosphorylation of downstream LRRK2 substrates [33, 34]; these alterations paralleled impairments in motor behaviour [34]. Future work to understand the interplay between different genetic alterations, LRRK2 signalling, and clinical phenotype will undoubtedly reveal fascinating biological insights [33].

A novel aspect of this study was the attempt to gauge the clinical severity of our monogenic PD cases against a backdrop of a large local cohort of unselected PD patients managed and evaluated by the same neurologist, using a standardised rating scale. CISI-PD ratings were incorporated into routine clinic consultations and typically took $\leq 10$ min. This facilitated inclusion of patients across the entire disease spectrum, including those with severe disability which studies tend to under-represent due to patient unwillingness or inability to participate [35]. The relative brevity of the instrument also helped to minimise data noncapture due to clinician time constraints - an important 
consideration particularly in under-resourced settings $[6$, 36]. The very wide ranges in subjects' ages, disease durations, CISI-PD scores, and LEDDs obtained in this study demonstrated the feasibility of applying the CISI-PD broadly, thereby minimising recruitment bias. These data provided supportive evidence of better-preserved cognition in the R1441C patients compared to "overall" PD patients, as seen in Figure 1b. An important caveat when making comparisons of global disease severity in these patients is the fact that both (vs. only approx. 9\% of the overall cohort) had undergone functional neurosurgical treatment for motor complications, which would tend to result in improvements in CISI-PD items 1-3.

So far, a few independent founders for the R144C mutation have been reported in European populations [11, 27]. To our knowledge, there have been only 2 reported cases of LRRK2 R1441C in patients of Asian ethnicity, both Han Chinese from Singapore and mainland China [10-14]. Interestingly, the haplotype in the LRRK2 region for our 2 patients would be consistent with a shared founder with the previously reported Singaporean Chinese patient based on ten identical single nucleotide polymorphisms (no data for rs10878246 available) $[9,11]$. The ancestors of Chinese people in Malaysia and Singapore (and other parts of South-East Asia) primarily migrated from Southern China beginning in the late 1700s [37]. Whether there is a common founder for Chinese R1441C PARK-LRRK2 cases is an area for future collaborative research.

This study has some limitations. Although the CISIPD has advantages in terms of facilitating broad recruitment of participants, it has yet to be widely adopted by the PD research community [38], hampering comparative assessments between studies [39]. CISI-PD scores are assigned through a subjective "global impression" of each aspect evaluated, and the original papers did not specify for patients to be rated in either the ON- or OFF-medication state. For item 1 (motor signs), we rated patients primarily according to what was observed in the clinic, but consideration was also given to historical information (e.g., if OFF periods were reported to occupy a large portion of the day and caused significant motor impairment, but the patient was only observed in the $\mathrm{ON}$-medication state, the rater would attempt to reconcile these states to best reflect the patient's condition as a whole).

In conclusion, this report contributes to the scarce literature on R1441C PARK-LRRK2, providing insight into epidemiological aspects and natural history. With LRRK2-targeted therapeutics now taking centre stage in disease-modification efforts in PD, work is urgently needed to further characterise the epidemiology, clinical correlates, and pathophysiology of the different LRRK2 mutations and variants.

\section{Acknowledgments}

S.-Y.L. is grateful to Prof. Pablo Martinez-Martin for his helpful comments regarding the CISI-PD.

\section{Statement of Ethics}

The study was approved by the Medical Research Ethics Committees, University of Malaya Medical Centre (UMMC; ID No. 732.5 and 20191010-7917) and Ministry of Health Malaysia (ID No. NMRR-10-935-7179 and NMRR-19-3762-52429) and conducted ethically in accordance with the World Medical Association Declaration of Helsinki. Written informed consent was obtained from all subjects.

\section{Disclosure Statement}

S.-Y.L., C.K., and K.L. have received honoraria from the Global Parkinson's Genetics Project (GP2) funded by the Michael J. Fox Foundation (MJFF) and the Aligning Science Across Parkinson's (ASAP) initiative. S.-Y.L. also reports lecturing honoraria from Medtronic, Lundbeck, Novartis, and UCB, and consultation fees from Lundbeck. P.B. is an employee, and A.R. the founder and CEO, of Centogene AG. The other authors have no conflicts of interest.

\section{Funding Sources}

S.-Y.L. and A.H.T. are supported by the University of Malaya Parkinson's Disease and Movement Disorder Research Program (PV035-2017). A.A.-A. is supported by a grant from the Ministry of Education Malaysia (FRGS/1/2018/SKK08/UM/02/3). C.K. and K.L. are supported by the DFG (FOR2488).

\section{Author Contributions}

Conception and design of the study: S.-Y.L., A.A.-A., C.K., K.L., A.H.T. Acquisition of data: S.-Y.L., J.L.L., A.H.T., A.A.-A., K.L., C.K., P.B., A.R., Y.W.T., K.B.L., K.A.M, Y.L.S. Analysis and interpretation of data: S.-Y.L., A.A.-A., K.L., K.B.L., A.H.T., C.K., Y.W.T. Drafting of manuscript: S.-Y.L. Revising the manuscript for intellectual content: S.-Y.L., K.L., A.A.-A., C.K., A.H.T., Y.L.S. Final approval of the manuscript to be submitted: all authors.
Lim et al. 


\section{References}

1 Blauwendraat C, Nalls MA, Singleton AB. The genetic architecture of Parkinson's disease. Lancet Neurol. 2020 Feb;19(2):170-8.

2 Domingo A, Klein C. Genetics of Parkinson disease. Handb Clin Neurol. 2018;147:21127.

3 Sardi SP, Cedarbaum JM, Brundin P. Targeted therapies for Parkinson's disease: from genetics to the clinic. Mov Disord. 2018 May; 33(5):684-96.

4 Tolosa E, Vila M, Klein C, Rascol O. LRRK2 in Parkinson disease: challenges of clinical trials. Nat Rev Neurol. 2020 Feb;16(2):97-107.

5 Peeraully T, Tan EK. Genetic variants in sporadic Parkinson's disease: east vs. West. Parkinsonism Relat Disord. 2012 Jan;18 Suppl 1:S63-5.

6 Lim SY, Tan AH, Ahmad-Annuar A, Klein C, Tan LC, Rosales RL, et al. Parkinson's disease in the Western Pacific Region. Lancet Neurol. 2019 Sep;18(9):865-79.

7 Shu L, Zhang Y, Sun Q, Pan H, Tang B. A comprehensive analysis of population differences in LRRK2 variant distribution in Parkinson's disease. Front Aging Neurosci. 2019 Jan;11:13.

8 Gopalai AA, Lim SY, Chua JY, Tey S, Lim TT, Mohamed Ibrahim N, et al. LRRK2 G2385R and R1628P mutations are associated with an increased risk of Parkinson's disease in the Malaysian population. BioMed Res Int. 2014; 2014:867321

9 Tan MM, Malek N, Lawton MA, Hubbard L, Pittman AM, Joseph T, et al. Genetic analysis of Mendelian mutations in a large UK population-based Parkinson's disease study. Brain. 2019 Sep;142(9):2828-44.

10 Tan EK, Skipper L, Chua E, Wong MC, Pavanni $\mathrm{R}$, Bonnard $\mathrm{C}$, et al. Analysis of 14 LRRK2 mutations in Parkinson's plus syndromes and late-onset Parkinson's disease. Mov Disord. 2006 Jul;21(7):997-1001.

11 Haugarvoll K, Rademakers R, Kachergus JM, Nuytemans K, Ross OA, Gibson JM, et al. Lrrk2 R1441C parkinsonism is clinically similar to sporadic Parkinson disease. Neurology. 2008 Apr;70(16 Pt 2):1456-60.

12 Peng F, Sun YM, Chen C, Luo SS, Li DK, Wang YX, et al. The heterozygous R1441C mutation of leucine-rich repeat kinase 2 gene in a Chinese patient with Parkinson disease: a five-year follow-up and literatures review. J Neurol Sci. 2017 Feb;373:23-6.

13 Trinh J, Zeldenrust FM, Huang J, Kasten M Schaake S, Petkovic S, et al. Genotype-phenotype relations for the Parkinson's disease genes SNCA, LRRK2, VPS35: MDSGene systematic review. Mov Disord. 2018 Dec;33(12): 1857-70.

14 MDSGene website (https://www.mdsgene org), accessed 27 March 2020.
15 Vollstedt EJ, Kasten M, Klein C, Aasly J, Adler C, Ahmad-Annuar A, et al.; MJFF Global Genetic Parkinson's Disease Study Group. Using global team science to identify genetic parkinson's disease worldwide. Ann Neurol. 2019 Aug;86(2):153-7.

16 Ben-Joseph A, Marshall CR, Lees AJ, Noyce AJ. Ethnic variation in the manifestation of Parkinson's disease: a narrative review. J Parkinsons Dis. 2020;10(1):31-45.

17 Klein C, Hattori N, Marras C. MDSGene: closing data gaps in genotype-phenotype correlations of monogenic Parkinson's disease. J Parkinsons Dis. 2018;8 s1:S25-30.

18 Lees AJ, Hardy J, Revesz T. Parkinson's disease. Lancet. 2009 Jun;373(9680):2055-66.

19 Martínez-Martín P, Forjaz MJ, Cubo E, Frades B, de Pedro Cuesta J; ELEP Project Members. Global versus factor-related impression of severity in Parkinson's disease: a new clinimetric index (CISI-PD). Mov Disord. 2006 Feb;21(2):208-14.

20 Martínez-Martín P, Rojo-Abuin JM, Rodríguez-Violante M, Serrano-Dueñas M, Garretto N, Martínez-Castrillo JC, et al. Analysis of four scales for global severity evaluation in Parkinson's disease. NPJ Parkinsons Dis. 2016 May;2(1):16007.

21 Martínez-Martín P, Rodríguez-Blázquez C, Mario Alvarez, Arakaki T, Arillo VC, Chaná P, et al. Parkinson's disease severity levels and MDSUnified Parkinson's Disease Rating Scale. Parkinsonism Relat Disord. 2015 Jan;21(1):50-4.

22 Low SC, Tan AH, Lim SY. Teaching Video NeuroImages: purposeless groaning in progressive supranuclear palsy. Neurology. 2017 Jan;88(1):e9.

23 Mainka T, Balint B, Gövert F, Kurvits L, van Riesen $C$, Kühn AA, et al. The spectrum of involuntary vocalizations in humans: A video atlas. Mov Disord. 2019 Dec;34(12):1774-91.

24 Lim SY, Tan AH, Lim JL, Ahmad-Annuar A Purposeless groaning in Parkinson's disease. J Mov Disord. 2018 May;11(2):87-8.

25 Tan AH, Hew YC, Lim SY, Ramli NM, Kamaruzzaman SB, Tan MP, et al. Altered body composition, sarcopenia, frailty, and their clinico-biological correlates, in Parkinson's disease. Parkinsonism Relat Disord. 2018 Nov;56:58-64.

26 Zainudin S, Daud Z, Mohamad M, Tan AT, Wan Mohamed WM. A summary of the Malaysian Clinical Practice Guidelines on Management of Obesity 2004. J ASEAN Fed Endoc Soc. 2011 Nov;26(2):101-4

27 Criscuolo C, De Rosa A, Guacci A, Simons EJ, Breedveld GJ, Peluso S, et al. The LRRK2 R1441C mutation is more frequent than G2019S in Parkinson's disease patients from southern Italy. Mov Disord. 2011 Aug;26(9): 1733-6.
28 de Oliveira LM, Barbosa ER, Aquino CC Munhoz RP, Fasano A, Cury RG. Deep brain stimulation in patients with mutations in Parkinson's disease-related genes: a systematic review. Mov Disord Clin Pract (Hoboken). 2019 Jun;6(5):359-68.

29 Artusi CA, Dwivedi AK, Romagnolo A, Pal G, Kauffman M, Mata I, et al. Association of subthalamic deep brain stimulation with motor, functional, and pharmacologic outcomes in patients with monogenic Parkinson disease: a systematic review and metaanalysis. JAMA Netw Open. 2019 Feb; 2(2):e187800.

30 Oosterveld LP, Allen JC Jr, Reinoso G, Seah SH, Tay KY, Au WL, et al. Prognostic factors for early mortality in Parkinson's disease. Parkinsonism Relat Disord. 2015 Mar;21(3):22630.

31 Silverdale MA, Kobylecki C, Kass-Iliyya L, Martinez-Martin P, Lawton M, Cotterill S, et al.; UK Parkinson's Pain Study Collaboration. A detailed clinical study of pain in 1,957 participants with early/moderate Parkinson's disease. Parkinsonism Relat Disord. 2018 Nov;56:27-32.

32 Hassan A, Wu SS, Schmidt P, Simuni T, Giladi N, Miyasaki JM, et al. The profile of longterm Parkinson's disease survivors with 20 years of disease duration and beyond. J Parkinsons Dis. 2015;5(2):313-9.

33 Alessi DR, Sammler E. LRRK2 kinase in Parkinson's disease. Science. 2018 Apr;360(6384): 36-7.

34 Xenias HS, Chen C, Kang S, Shanmugasundaram B, Cherian S, Situ X, et al. Pathogenic LRRK2 R1441C mutation is associated with striatal alterations. bioRxiv. 2020. doi:https:// doi.org/10.1101/2020.03.11.986455.

35 Agarwal S, Fleisher JE. Reaching those most in need: a call to action for advanced Parkinson's disease. Eur Neurol Rev. 2016;11(1):201.

36 Lim SY, Tan AH, Fox SH, Evans AH, Low SC Integrating patient concerns into Parkinson's disease management. Curr Neurol Neurosci Rep. 2017 Jan;17(1):3.

37 Lockard CA. Chinese migration and settlement in Southeast Asia before 1850: making fields from the sea. HIst Compass. 2013 Sep; 11(9):765-81.

38 Malek N, Swallow DM, Grosset KA, Lawton MA, Marrinan SL, Lehn AC, et al.; PRoBaND. Tracking Parkinson's: study design and baseline patient data. J Parkinsons Dis. 2015;5(4): 947-59.

39 Lim SY, Tan AH. Historical perspective: the pros and cons of conventional outcome measures in Parkinson's disease. Parkinsonism Relat Disord. 2018 Jan;46 Suppl 1:S47-52. 